\title{
Traffic and Storage of Metal Ions
}

\section{P301}

\section{How NikR from Helicobacter pylori recognizes DNA:} metal ion requirements and sequence specificity Sarah L. J. Michel, Nuvjeevan S. Dosanjh, Abby L. West Department of Pharmaceutical Sciences, University of Maryland, School of Pharmacy, Baltimore, MD 21201, USA.

smichel@rx.umaryland.edu

$H P$ NikR is a prokaryotic nickel binding transcription factor found in Helicobacter pylori where it regulates the expression of multiple genes including those involved in acid adaptation and nickel ion homeostasis. Of particular importance is HPNikR's role in the regulation of the nickel dependent enzyme urease which is critical for the organism's survival in its environmental niche-the acidic gastric epithelium. The target operator sequences of the genes regulated by $H P$ NikR do not contain identifiable palindromes and the exact mechanism(s) by which $H P$ NikR recognizes DNA is unknown. A direct and competitive fluorescence anisotropy (FA) assay has been developed to probe both the metal ion requirements and sequence specificity of $H P N i k R$ for $P$ ureA, the operator sequence for the urease gene. These studies have revealed that apo- $H P$ NikR does not bind to $P$ ureA while Ni(II)HPNikR binds $P$ ureA with nanomolar affinity, but only in the presence of a second metal ion [magnesium, calcium or manganese(II)] suggesting that HPNikR contains a second, lowaffinity metal binding site. Removal of a loosely conserved 'putative' palindrome sequence in the PureA operator abrogates $H P$ NikR binding. Studies examining the recognition properties of a series of other genes directly regulated by $H P$ NikR also show a requirement for a second metal ion coupled with a two-tiered mode of DNA recognition. Together, we propose a model of $H P N i k R / D N A$ binding wherein specific metal ions must be coordinated to high and low affinity sites to modulate binding.

\section{References}

1. Dosanjh NS, West AL, Michel SLJ (2009) Biochemistry 48:527536

\section{P302}

\section{A novel vanadium transporter of the Nramp/DCT} family from a vanadium-rich Ascidian Ascidia sydneiensis samea

Tatsuya Ueki ${ }^{1}$, Nobuaki Furuno ${ }^{2}$, Hitoshi Michibata ${ }^{1}$

${ }^{1}$ Department of Biology Science

${ }^{2}$ Amphibian Institute, Graduate School of Science, Hiroshima University, Hiroshima 739-8526, Japan. ueki@ hiroshima-u.ac.jp Vanadium is one of essential transition metals in biological systems. Several key proteins such as vanadium-binding proteins (Vanabins) related to vanadium accumulation have been isolated, but transporter for vanadium ions has not been identified [1]. Here we report the identification and cloning of a Nramp/DCT family of membrane metal transporter (AsNramp) from an ascidian Ascidia sydneiensis samea, which can accumulate extremely high levels of vanadium in the vacuoles of signet ring cells, which are one type of blood cell called

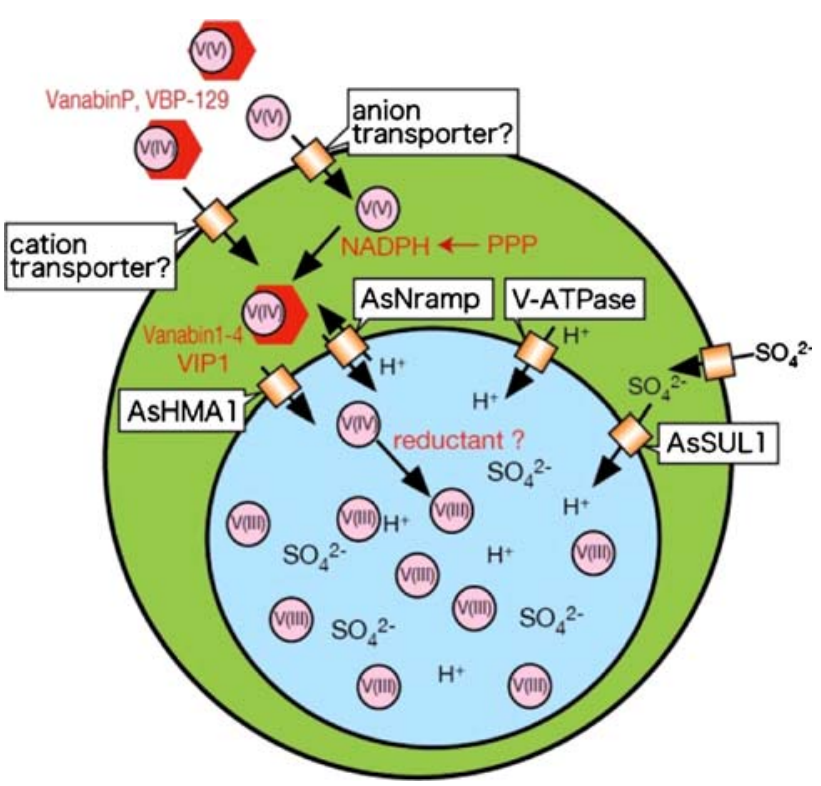

Fig. 1 A model of vanadium transport pathway and related membrane transporters in vanadocytes

vanadocytes. By using Xenopus oocyte expression system, we revealed that AsNramp transported $\mathrm{VO}^{2+}$ into oocyte at $\mathrm{pH}>6$, while no significant activity was observed at $\mathrm{pH}<6$. Sodium ions inhibited the AsNramp-dependent transport of $\mathrm{VO}^{2+}$. Western blot analysis showed that $A s$ Nramp was localized on the vacuolar membrane of vanadocytes. These results indicated that $A s \mathrm{Nramp}$ is a novel $\mathrm{VO}^{2+} / \mathrm{H}^{+}$antiporter. We propose that $A s$ Nramp mediates vanadium accumulation coupled with the electrochemical gradient generated by vacuolar $\mathrm{H}^{+}$-ATPase (Fig. 1).

\section{References}

1. Michibata H et al (2007) ACS Symp Ser 974:264-280

\section{P303}

\section{Stress-regulated transportation by polypyridyl} ruthenium complexes in $E$. Coli. discovered by metalloprotomics

Chien-Chung Cheng, Ming-Yi Ho, Min-Lun Chiou

Department of Applied Chemistry, National Chia-Yi University, Chia-Yi City, Taiwan 60004, ROC. cccheng@mail.ncyu.edu.tw Transportation of metal complexes into the target cell is important for the development of metallopharmaceuticals. Monochlororuthenium complexes, $\mathrm{Ru}($ terpy $)(\mathrm{NN}) \mathrm{Cl}^{+}(\mathrm{NN}$, bidentate ligand) were demonstrated in the structure-reactivity relationship in the bacterial and the inhibition of zebrafish growth. It will be interesting to understand how to transport ruthenium complexes into cells to induce the cell-growth 
inhibition. The total proteins distribution was analyzed by twodimensional gel electrophoresis and ESI-/MALDI-MS. Based on the protein identification, the outer membrane proteins of $E$. Coli. were found to play an important role in the transportation of $\mathrm{Ru}(\mathrm{II}) \mathrm{com}$ plexes. $O m p F$ is considered as a major target protein, which is one of osmolarity response proteins. The intensity of outer membrane proteins is varied by the concentration of $\mathrm{Ru}(\mathrm{II})$ complexes and $10 \%$ sucrose solution. Its interaction with transition metal complexes does not disclose yet. The interaction of over-expressed ompF protein with different $\mathrm{Ru}(\mathrm{II})$ complexes monitored by electronic absorption spectra and circular dichroism will be discussed.

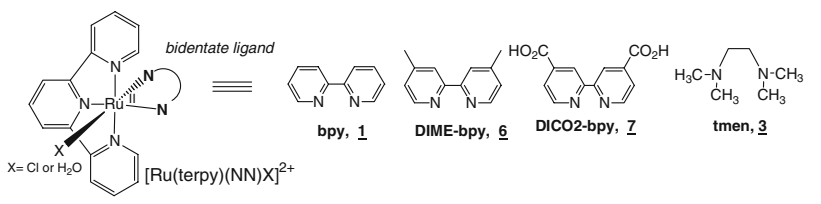

\section{P304}

\section{Fluorescence of a Cu-accumulator moss Scopelophila cataractae}

Hiromitsu Nakajima, Kiminori Itoh

Graduate School of Engineering, Yokohama National University, Yokohama 240-8501, Japan. h-nakaji@ynu.ac.jp

Scopelophila cataractae (Fig. 1) is known as a "copper moss" and is reported to have great potential to treat $\mathrm{Cu}$-polluted water. However, the mechanism of $\mathrm{Cu}$ accumulation by this moss remains unclear.

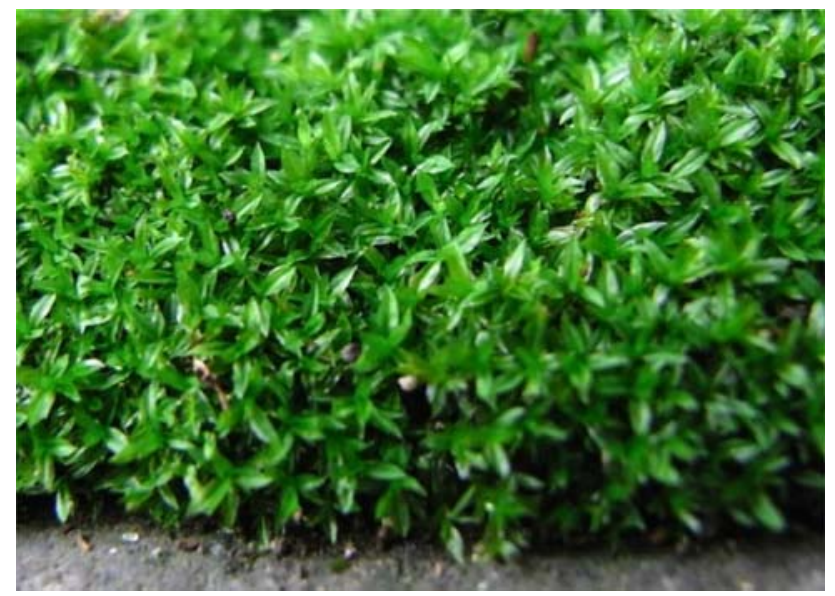

Fig. 1 Scopelophila cataractae

Optical spectroscopy could be an efficient method to obtain information needed to clarify the mechanism because the color of $S$. cataractae is deep green, deeper than those of other common mosses, and its color must reflect the structure and photoreaction related to $\mathrm{Cu}$ accumulated. Nevertheless, the photoabsorption and fluorescence have not been studied for S. cataractae, as far as we know. Even for mosses spectral data are limited. Hence, we studied the fluorescence of $S$. cataractae and several common mosses, and found that the fluorescence of chlorophyll for $S$. cataractae increases remarkably after moistening whereas those for the other mosses increase slightly; this remarkable increase is suggested to be characteristic of $S$. cataractae. To elucidate the relationship between the increase in fluorescence and the $\mathrm{Cu}$ accumulation, their chlorophyll and $\mathrm{Cu}$ concentrations are compared. Based on the results, the relationship and the mechanism of $\mathrm{Cu}$ accumulation by $S$. cataractae are discussed.

\section{P305}

Effect of the $\mathbf{N}$-terminus of the human high affinity copper transporter 1 (hCtr1) on cisplatin hydrolysis and uptake

Xinghao Wang, Xiubo Du, Hongyan Li, Denise S. B. Chan,

\section{Hongzhe Sun}

Department of Chemistry and Open Laboratory of Chemical Biology, The University of Hong Kong, Pokfulam Road, Hong Kong, People's Republic of China.wangmouse1985@gmail.com

Cisplatin(cis-diamminedichloridoplatinum(II), cis-DDP) and other platinum complexes, such as carboplatin and oxaliplatin, have been widely used in the chemotherapy of different types of cancer [1]. By forming adducts with DNA, they can inhibit DNA replication and cause cell death. The entrance pathway of cisplatin was originally thought to be passive diffusion. However, recent in vivo and in vitro studies indicated that human copper transporter 1 (hCtr1) — an integral membrane protein necessary for high affinity copper uptake — could also be a transporter for cisplatin and other platinum complexes [2]. Yet, the mechanism of interaction between cisplatin and hCtrl at the molecular level is currently unclear. In this work, we have successfully overexpressed, purified and characterized the $\mathrm{N}$-terminal extracellular domain of hCtr1 (hCtr1_ $N$, residues $1-55$ ), which contains 2 methionine-rich and 2 histidine-rich regions. A small tag (GB1 with $8 \mathrm{kD})$ with no metalbinding-site is attached to hCtrl_ $N$ to improve its solubility and stability. ${ }^{1} \mathrm{H}$ and $2 \mathrm{D}\left[{ }^{1} \mathrm{H},{ }^{15} \mathrm{~N}\right] \mathrm{HSQC}$ NMR experiments of ${ }^{15} \mathrm{~N}$-labeled cisplatin and tagged hCtr1_ $N$ were carried out to examine the effect of the protein on cisplatin hydrolysis. Our results show that cisplatin hydrolyses rapidly by binding methionine residues of tagged hCtr1_ $N$, following by the quickly loss of its ammonia ligand. Both NMR and ESI-MS results prove that the mutation of hCtr1_ $N$ methionine to alanine could reduce the effect of protein on cisplatin hydrolysis and, especially, the loss of ammonia ligand. The gel filtration work shows that cisplatin can cause oligomerization of protein, probably due to the cross-linking of the proteins by the anticancer drug.

This work was supported by Research Grants Council of Hong Kong (HKU7512/05 M, HKU7043/06P, HKU2/06C, HKU1/07C, HKU7038/08P), National Science Foundation of China and the University of Hong Kong.

\section{References}

1. Wang D, Lippard SJ (2005) Nat Rev Drug Discov 4:307-320

2. Ishida S, Lee J, Thiele SJ, Herskowitz I (2002) Proc Natl Acad Sci USA 99:14298-14302

\section{P306}

Identification of mineral forms in plants: an approach by multi-nuclear NMR spectroscopy

Takashi Iwashita ${ }^{1}$, Daisei Ueno ${ }^{2}$, Renfang Shen ${ }^{2}$, Jian Feng $\mathrm{Ma}^{2}$ ${ }^{1}$ Suntory Institute for Bioorganic Research, Wakayamadai 1-1-1, Shimamoto-cho, Osaka 618-8503, Japan,

${ }^{2}$ Research Institute for Bioresources, Okayama University, Chuo 2-20-1, Kurashiki 710-0046, Japan. iwashita@sunbor.or.jp Metals like aluminium and cadmium are usually toxic for plants at higher concentrations, but some plants have developed mechanisms for detoxifying these metals. Some plants known as hyperaccumulator are able to accumulate extraordinarily high concentrations of these metals. For example, buckwheat accumulates high $\mathrm{Al}$ in the leaves and Thlaspi caerulrscens (Ganges ecotype) accumulates high $\mathrm{Cd}$. To understand the tolerance mechanism, identification of the chemical form of these metals in plants is very important. ${ }^{27} \mathrm{Al}$ has a spin quantum number $5 / 2$, while ${ }^{113} \mathrm{Cd}$ has a spin quantum number $1 / 2$, showing sharp peak in NMR spectrum. Investigation with ${ }^{27} \mathrm{Al}-\mathrm{NMR}$ showed that the 
chemical shift of ${ }^{27} \mathrm{Al}$ in the buckwheat leaves with low $\mathrm{Al}$ concentration was $16.1 \mathrm{ppm}$, which corresponds to Al-oxalate complex, a nontoxic $\mathrm{Al}$ form. However, at higher $\mathrm{Al}$ concentrations, both $\mathrm{Al}$-oxalate and $\mathrm{Al}-$ citrate were detected. By using ${ }^{113} \mathrm{Cd}-\mathrm{NMR}$, the $\mathrm{Cd}$ form was identified as $\mathrm{Cd}-$ malate complex in T. caerulescens leaves and ionic $\mathrm{Cd}$ in the xylem sap in Arabudopsis halleri. These results indicate that NMR method is useful to identify chemical forms of minerals in plants.

\section{References}

1. Shen R, Iwashita T, Ma JF (2004) J Exp Bot 55:131-136

2. Ueno D, Iwashita T, Zhao FJ, Ma JF (2008) Plant Cell Physiol 49:540-548

\section{P307}

Characterisation of TcHMA4, a Cd/Zn ATPase purified from roots of the hyperaccumulator plant Thlaspi

\section{caerulescens}

Barbara Leitenmaier ${ }^{1}$, Anastasia Stemke ${ }^{1}$,

Wolfram Meyer-Klaucke ${ }^{2}$, Hendrik Küpper ${ }^{1,3}$

${ }^{1}$ Universität Konstanz, Fachbereich Biologie, Universitätsstr.10,

78457 Constance, Germany.

${ }^{2}$ EMBL Outstation Hamburg c/o Deutsches Elektronen-Synchrotron, Notkestrasse 85, 22603 Hamburg, Germany.

${ }^{3}$ Faculty of Biological Sciences and Institute of Physical Biology, University of South Bohemia, Branišovská 31, 37005 České

Budejovice, Czech Republic.

barbara.leitenmaier@uni-konstanz.de

TcHMA4 belongs to the family of $\mathrm{P}_{1 \mathrm{~b}}$-type ATPases, which are known from genomics to occur in all organisms from bacteria to plants and animals, but their protein biochemistry is still largely unknown. TcHMA4 transports zinc as well as cadmium over the cytoplasmic membrane. We isolated it from natural overexpression in roots of the $\mathrm{Cd} / \mathrm{Zn}$ hyperaccu-

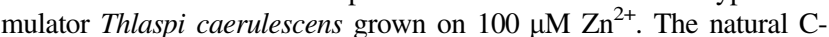
terminal His tag of TcHMA4 consisting of 9 histidines was used for purification via metal affinity chromatography and it was found that the natural form of this transporter is modified by post-translational processing as SDS gels and western blots did not show the full size protein that was predicted from the cDNA sequence. After reconstitution in artifical lipid vesicles, ATPase activity tests of the purified protein showed that it is in its active state and its activity can be strongly increased by the addition of various metals. Further, EXAFS measurements revealed that the ligands of TcHMA4 involved in the binding of cadmium are mainly cysteins (according to the sequence, 58 cys residues are present in TcHMA4), histidines are only little involved in cadmium binding. Currently we are further characterising the binding and transport of different heavy metals by purified TcHMA4 in order to elucidate the metal specificity as well as the number and affinity of different binding sites. Currently we are working on transport studies using specific fluorescent dyes.

\section{P308}

\section{Characterization of a Copper(I) Bridged Intermediate} between Wilson Disease Protein Domain 4

\section{and Its Copper Chaperone, HAH1}

Ivano Bertini $^{1}$, Francesca Cantini ${ }^{1}$, Simone Ciofi-Baffoni ${ }^{1}$, David Huffman ${ }^{2}$, Brian Zeider ${ }^{2}$

${ }^{1}$ Center for Magnetic Resonance (CERM), University of Florence, Sesto Fiorentino 50019, Italy. ${ }^{2}$ Department of Chemistry, Western Michigan University, Kalamazoo, Michigan 49008, USA.

brian.a.zeider@wmich.edu

Copper is scrupulously regulated within the cell to maintain proper balance of redox activity and storage. One protein involved in this process is Wilson disease protein, which is responsible for moving copper into vesicles for either storage or removal from the cell. Previous work has shown copper is transferred to the Wilson disease protein via its metallochaperone, HAH1 [1-3]. The proposed mechanism for this transfer seems to involve a transient 3-coordinate copper(I) intermediate. Similar work done on Saccharomyces cerevisiae has shown that a 3-coordinate intermediate could exist between Atx1 (analog of HAH1) and its partner Ccc2 [4]. Trapping this intermediate involved selective mutation of individual cysteines involved in the copper(I) binding. NMR titrations were done to look for pertinent shifts in the chemical environment around the copper(I) binding site, as well as mobility changes between bound and unbound forms, indicating the presence of the bridged structure.

\section{References}

1. Yatsunyk L, Rosenzweig A (2007) J Biol Chem 282:8622-8631

2. Achila D, Banci L, Bertini I, Bunce J, Ciofi-Baffoni S, Huffman D (2006) PNAS 103:5729-5734

3. Banci L, Bertini I, Cantini F, Massagni C, Migliardi M, Rosato A (2009) J Biol Chem (Accepted)

4. Banci L, Bertini I, Cantini F, Felli I, Gonnelli L, Hadjiliadis N, Pierattelli R, Rosato A, Voulgaris P (2006) Nat Chem Biol (2006) $2: 367-368$

\section{P309}

\section{Copper-thioneins form sulphide-rich} cadmium-complexes both recombinantly and natively S. Atrian', M. Capdevila ${ }^{2}$

${ }^{1}$ Departamento de Genética, Facultad de Biología, Universitat de Barcelona, 08028 Barcelona, Spain.

${ }^{2}$ Departamento deQuímica, Facultad de Ciencias, Universitat Autònoma de Barcelona, 08193 Bellaterra, Spain. satrian@ub.edu Metallothioneins (MTs) are ubiquitous, small cysteine-rich, metalbinding proteins [1]. We have recently shown that MTs can be classified following a sound stepwise gradation between extreme zinc- and extreme copper-thioneins [2]. When strict $\mathrm{Cu}$-thioneins are recombinantly synthesized in $\mathrm{Cd}(\mathrm{II})$-rich bacterial cultures, they yield a mixture of species of different stoichiometry with a significant presence of $\mathrm{S}^{2-}$ ligands in their metal-complexes [3]. In this work we aimed at studying the recombinant $\mathrm{Cd}(\mathrm{II})-\mathrm{Cup} 1$ complexes, being Cup1 the most extreme and paradigmatic Cu-thionein [2]. But, most significantly, we also addressed a long-standing question: are the $\mathrm{S}^{2-}$ ligands in metal-MT complexes a mere consequence of heterologous synthesis, or are they also present in native complexes. To this end, we purified native $\mathrm{Cd}(\mathrm{II})$-Cup1 produced by the mutant $301 \mathrm{~N} \mathrm{~S}$. cerevisiae strain, where a high Cup1 expression in front of an excess cadmium is feasible. (We thank Prof. D. Winge (Utah, USA) for providing us with this yeast strain, isolated in Prof. T. Murayama's lab (Matsuyama, Japan)). Our results show that recombinant and native syntheses yield spectroscopically- and spectrometrically equivalent $\mathrm{Cd}(\mathrm{II})-\mathrm{MT}$ preparations, comprising a subset of $\mathrm{S}^{2-}$-containing $\mathrm{Cd}(\mathrm{II})$-MT complexes. Further characterization of the $\mathrm{Cd}(\mathrm{II})$ complexes formed by several $\mathrm{Cu}$-thioneins is now in progress. It is also worth discussing how nature seems to avoid such complexes, either by extreme metal compartmentalization, or by strict promoter responsiveness.

\section{References}

1. Sigel A, Sigel H, Sigel RKO (eds) (2009) Metal ions in life sciences, vol 5. RSC, Cambridge

2. Bofill R, Capdevila M, Atrian S (2005) Metallomics. doi:10.1039/ b904953c (in press)

3. Capdevila M, Domenech J, Pagani A, Tio L, Villarreal L, Atrian S (2005) Angew Chem Int Ed 44:4618-4622 


\section{P310}

Influence on phagocytic activity of human neutrophils of thiazoline derivative ligands and their $\mathrm{Cu}$ (II)

\section{complexes}

F. J. Barros ${ }^{1}$, A. M. Lozano ${ }^{1}$, F. Luna ${ }^{1}$, E. Viñuelas ${ }^{1}$, A. Bernalte ${ }^{1}$, A. B. R. Moratinos ${ }^{2}$

${ }^{1}$ Department of Organic and Inorganic Chemistry, Extremadura University, 06071 Badajoz, Spain.

${ }^{2}$ Department of Fisiology, Extremadura University, 06071 Badajoz, Spain. fergaba@unex.es

Phagocytosis is one of the principal processes of the innate immune system. One type of phagocytes are the neutrophils which eliminates invading microorganisms or foreign materials by means of a specialized mechanism termed the respiratory burst [1]. Copper plays an important role in the development and maintenance of the immune system, its deficiency reduces both the number of neutrophils in human peripheral blood and their ability to generate superoxide anion and kill ingested microorganisms [2]. We have investigated the influence of the coordination to copper(II) ion of two thiazoline derivative ligands on the phagocytic activity of human neutrophils.

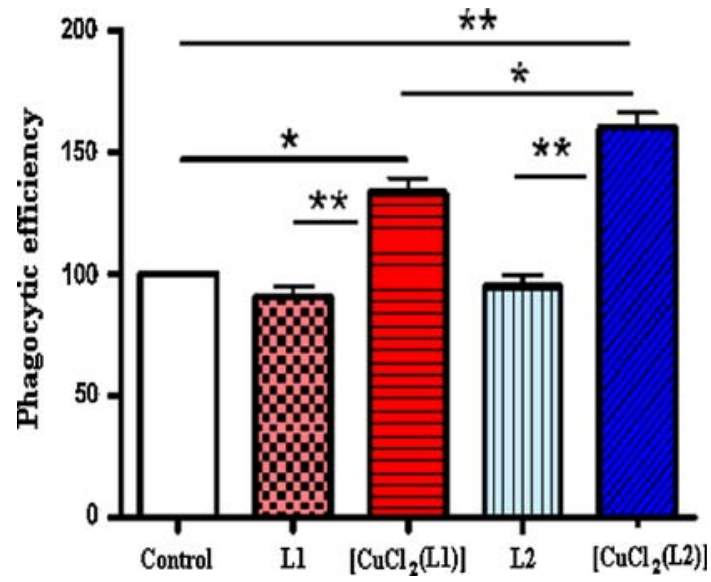

References

1. Muñoz M, Cedeño R, Rodríguez J, Van der Knapp WPW, Mialhe

$\mathrm{E}$, Bachère E (2000) Aquaculture 191:89-107

2. Percival SS (1998) Am J Clin Nutr 67:1064-1068

\section{P311}

\section{Redox dynamics of the iron in vivo after} the administration of ferric nitrilotriacetate Tetsuya Ozaki $^{1}$, Yoshie Asahara ${ }^{2}$, Teruyuki Kawabata ${ }^{1}$

${ }^{1}$ Department of Applied Physics, School of Science, Okayama University of Science, Okayama 700-0005, Japan.

${ }^{2}$ Medical Science Education Center, Okayama University of Science, Okayama 700-0005, Japan. kawabata@dap.ous.ac.jp

Ferric nitrilotriacetate [Fe(III)-nta] is a renal carcinogen through free radical mechanism and the $\mathrm{Fe}$ (III)-nta-injected animals are used as a model of free radical-induced injuries and carcinogenesis $[1,2]$. We also reported a new iron chelate, ferric iminidiacetate [Fe(III)-ida] similar to Fe(III)-nta [3]. It is believed in general that iron may be reduced to ferrous ion by some reductants, and through a Fenton-like reaction produce hydroxy and/or lipid peroxy radicals:

$\mathrm{Fe}(\mathrm{II})-\mathrm{nta}+\mathrm{H}_{2} \mathrm{O}_{2} \rightarrow \mathrm{Fe}(\mathrm{III})-\mathrm{nta}+\mathrm{OH}^{-}+\cdot \mathrm{OH}$

However, we do not know a dynamic change of the $\mathrm{Fe}(\mathrm{II}) / \mathrm{Fe}(\mathrm{III})$ ratio in vivo. This question is pivotal to understand iron-induced free radical injuries. We elucidated the ratio in the blood and kidneys after a single injection of $\mathrm{Fe}(\mathrm{III})$-nta into rats with UV-vis and ESR. $\mathrm{Fe}(\mathrm{III})$-nta injected into rat intraperitoneally was reduced and the half was in a ferrous state in the blood. Some mononuclear ionic iron(III) was demonstrated by ESR. Kidney was an organ with more reduced environment than blood, and most of the iron was in a reduced form, the structure of which was unknown because the iron is ESR-silent.

\section{References}

1. Okada S (2003) Biometals 16(1):99-101

2. Mizuno R, Kawabata T, Sutoh Y, Nishida Y, Okada S (2006) Biometals 19(6):675-683

\section{P312}

\section{Properties of cadmium(II) complex with macrocyclic} ligand

Mi Hyang Jeong, Jin Suk Gwag, Ju Chang Kim

Department of Chemistry, Pukyong National University, 599-1 Daeyeon 3-dong, Nam-gu, Busan 608-737, Korea. mhjeong@pknu.ac.kr

Cyclam and its derivatives are important compounds due to their antiHIV activity. It has been suggested that the carboxylate groups of aspartate residues Asp 171 and Asp 262 of the CXCR4 co-receptor play key roles on the recognition of cyclam moieties. Such interactions are likely to be highly dependent on cyclam configurations. In this presentation, we attempted reactions between $\mathrm{Cd}(\mathrm{L})\left(\mathrm{ClO}_{4}\right)_{2}$ $(\mathrm{L}=$ tetraazamacrocycle) and carboxylates to better understand the interaction between reactivity and cyclam conformations.

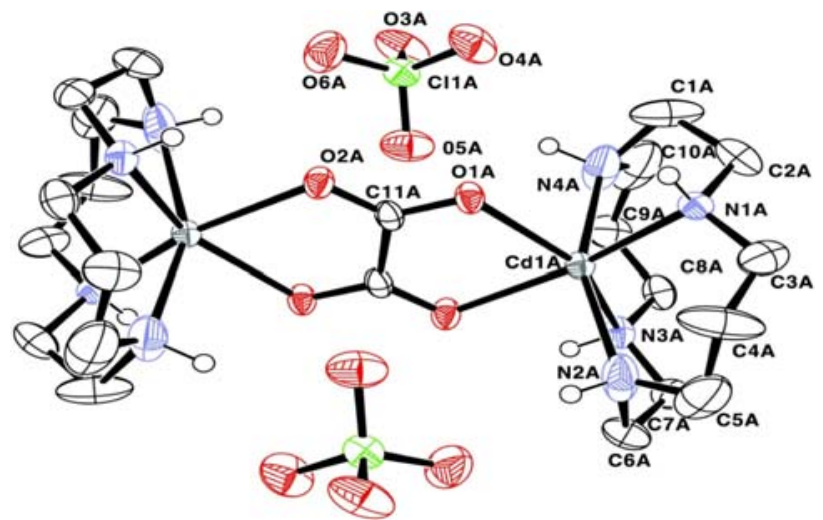

\section{References}

1. Gerlach LO, Jakobsen JS, Jensen KP, Rosenkilde MR, Skerlj RT, Ryde U, Bridger GJ, Schwartz TW (2003) Biochemistry 42:710-717 2. Liang X, Parkinson JA, Parsons S, Weishäupl M, Sadler J (2002) Inorg Chem 41:4539-4547 